\title{
NEONATAL SURGERY
}

\begin{abstract}
Introduction
In Singapore the majority of expectant mothers will have a fetal abnormality scan at around 18 weeks of fetal gestation. This is a very detailed scan, and in the following few months routine growth scans will be done. The fetal abnormality scan looks at various anatomical as well as physical parameters, and troubleshoots the fetus looking for suggestions or evidence of malformations. ${ }^{1,2}$ In our hospital around 14000 deliveries annually gives our maternal fetal physicians wide experience and excellent diagnostic skills.
\end{abstract}

ANETTE JACOBSEN

\section{Counscelling :}

The detection of an anomaly in the fetus triggers a series of events, which will impact the pregnancy positively. Neonatologist, cardiologist, surgeon as well as Obstetrician will counsel in detail to ensure optimal care for mother and child ${ }^{1}$.

The counseling will also ensure parents are fully prepared for eventual outcomes and events after delivery. Parents in discussion with the counseling team will have to make decisions of the optimal siting, timing and mode of delivery. For a child with suspected complex anomalies, a tertiary setting is preferred for optimal neonatal care. For a child with suspected surgical problems, delivery obviously is better done in a setting with neonatal surgical and anesthetic expertise. The majority of pregnancies are allowed to progress naturally to term. Fetus with a condition, which is incompatible with postnatal life, may be terminated after full disclosure, if this is legal and socially acceptable to parents.

\section{At Birth}

Neonatologists and surgeons will standby at delivery for infants with confirmed surgical anomalies. The neonatologist and surgeon will work together to fully

Correspondence to: Dr. Anette Jacobsen, Pediatric Surgeon, KK Hospital, Singapore evaluate the baby, and prepare for correctly timed further intervention.

As we know 'accidents' do not happen alone, a detailed repeat study of the baby including skeletal survey, renal and cardiac ultrasounds are included to exclude syndromes and associations. A chromosome analysis and genetic studies may be indicated. The Pediatric Radiologist is often involved in further detailed studies of the anatomy and of any anomalies. 3D CT reconstruction and MRI studies can give beautiful anatomical 'roadmaps' for the surgeon, and may help in the preparation for surgery. No surgery will take place without full evaluation of the infant and stabilization of the infant. The only exception may be in conditions such as a ruptured sacrococcygeal teratoma, where vascular control and control of hemorrhage is paramount to the survival of the baby.

\section{Neonatal surgical conditions.} Esophageal Atresia.

Antenatally, the $O+G$ specialists will see a dilated upper pouch and often will not see any stomach bubble, or the stomach bubble is very small. If in addition there is a decreased amount of liquor surrounding the baby, this is very suggestive of Esophageal atresia. Generally there is not problem taking these babies uneventfully to term and a normal delivery. But if not made antenatally, diagnosis must be made at birth before the child is fed. This prevents aspiration pneumonia from developing as the presenting complaint in a vomiting baby. Surgery for repair of Esophageal atresia will only take place after full evaluation to exclude associations such as VATER association. A plain chest $\mathrm{X}$ ray will identify a blind upper pouch and gas in the distal bowel identifying a fistula of the distal esophagus to the carina or above. (Figure 1.) Generally $85 \%$ of these patients will have primary repair through a right thoracotomy (Figure 2.), and the majority have a good prognosis. Long gap atresias and syndromic babies will have poorer prognosis. Complications include anastomotic leak, strictures and gastro-esophageal reflux. 


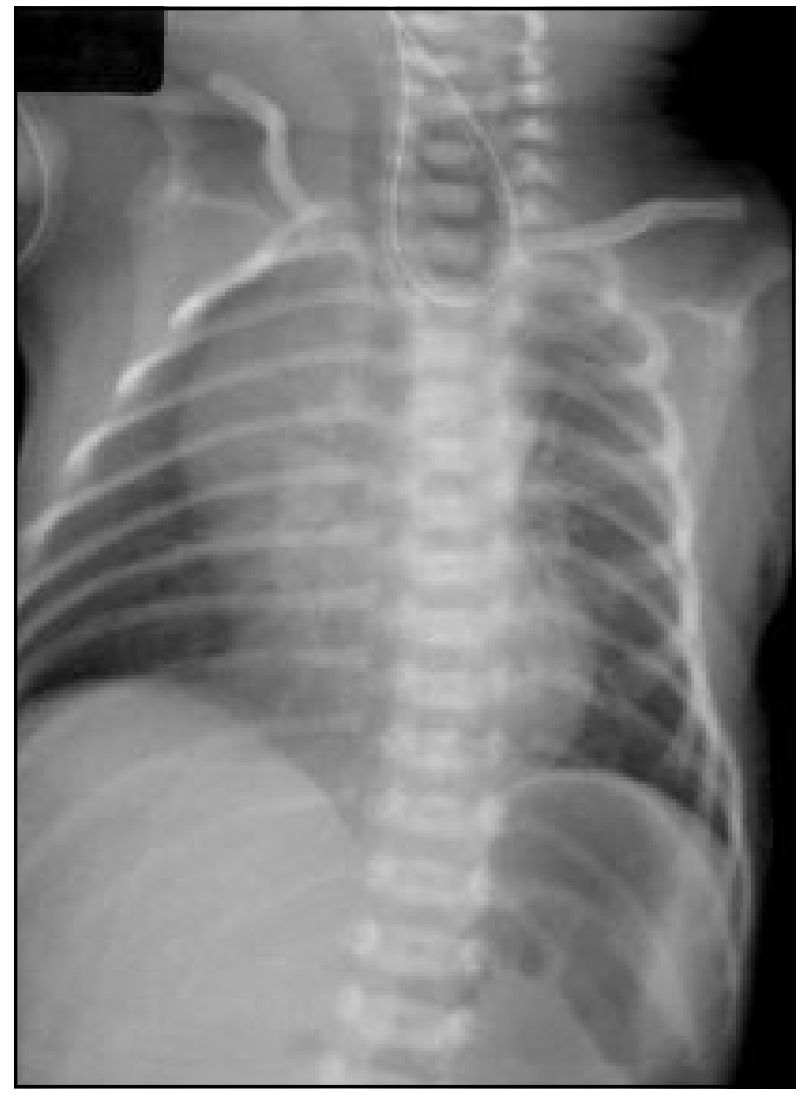

Fig.-1: Chest $X$ Ray showing a blind upper pouch with distal bowel gas indication presence of a tracheooesophageal fistula.

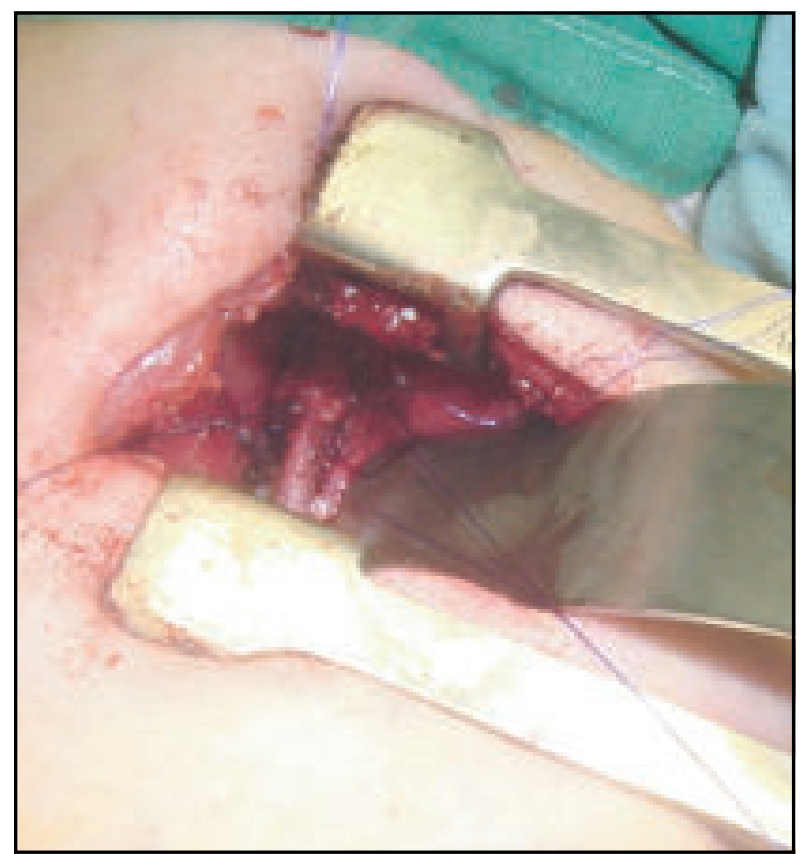

Fig.-2: Esophageal atresia repair through an extra plural right thoracotomy. There is a feeding tube crossing the anastomosis inside the esophagus.

\section{Congenital Diaphragmatic Hernia.}

In $\mathrm{CDH}$ the antenatal scan reveals loops of bowel most commonly in the left chest. The diaphragmatic defect is most commonly posterior with the spleen, stomach and large parts of small and large bowel residing in the left chest. This causes compression on the left lung with subsequent possible development of pulmonary hypoplasia. The pregnancy normally will go to term, and a normal delivery is standard. Designated centers in the US are performing fetal surgery for this condition, having attempted identification of a high risk case. We do not have this research protocol in Singapore, and at birth, the neonatologist will work very closely with the Pediatric Surgeon to achieve optimal timing of surgery.

The baby is stabilized, intubated and ventilated and an adequate sized nasogastic tube is inserted for continuous decompression of the bowel. (Figure 3) The ventilatory cum pulmonary status is carefully monitored, and often modifications in the ventilation to include permissive hyercapnoea as well as high frequency ventilation are required. Echmo is used in selected cases, and Magnesium Sulphate infusion and Nitric Oxide inhalation is also used to improve the pulmonary and ventilatory status.

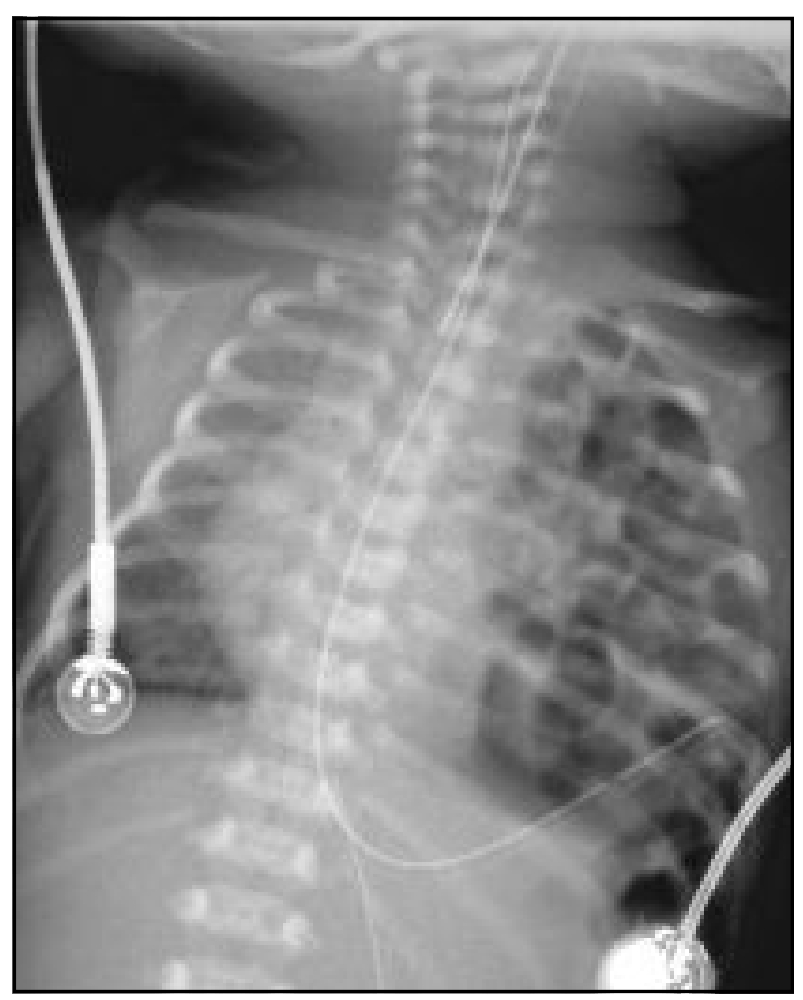

Fig.-3: Chest and abdominal $X$-ray in $C D H$. The nasogastric tube is in the stomach (which is in the chest with small and large bowel. 
The surgeons will only attempt repair of the diaphragm in a stable patient - sometimes after a period of stabilization up to $7-10$ days. A direct repair of the diaphragm after mobilization of the posterior lip of the diaphragm is possible in most cases (Figure 4.) If not an abdominal wall flap or a Gore-Tex patch can be used. Not all patients make it to surgery, the main problem being pulmonary hypertension and persistent fetal circulation. In those who come to surgery the prognosis is generally good with at least $80 \%$ survival. ${ }^{3,4,5}$

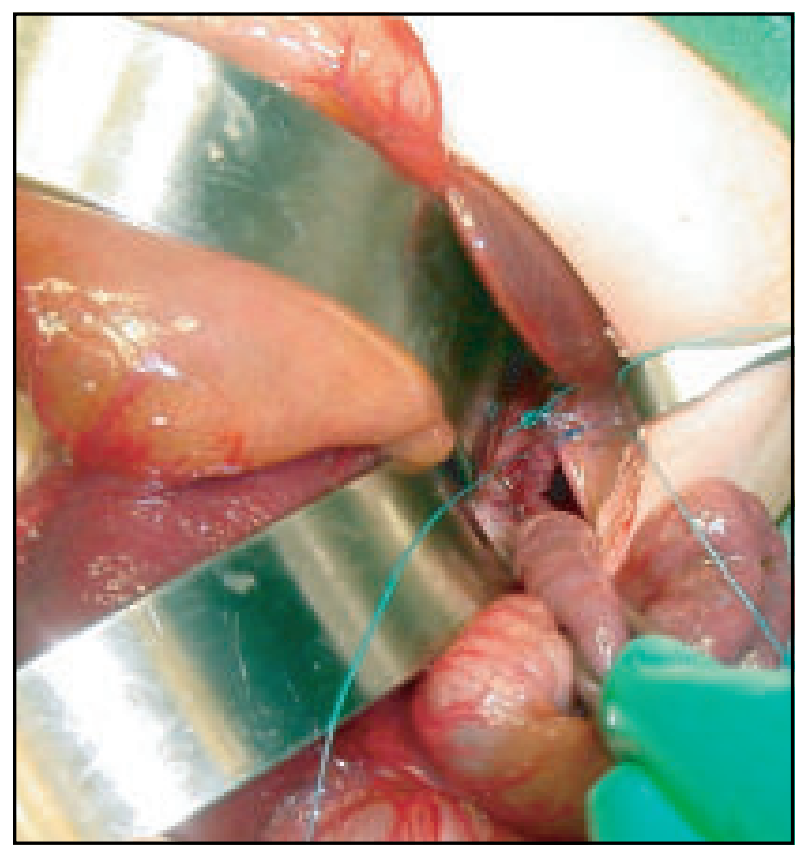

Fig.-4: Direct repair of the diaphragm after reduction of the contents into the abdomen. A non-absorbable suture is used.

\section{Bowel atresias.}

Duodenal and small bowel atresias are identified antenatally through 'double bubble' or triple / multiple bubbles' indication proximal dilated intestines. The mother may have polyhydramnios. The pregnancy is generally uneventful and is allowed to go to term, with normal delivery being the norm. In general a patient with duodenal atresia will need to be fully worked up for chromosomal abnormalities as well as other malformations and or syndromes. Small bowel atresias in general occur in isolation. In particular duodenal atresia may be associated with Down syndrome, where cardiac, renal and large bowel malformations also occur. (VU reflux, and Hirschprung's or Ano-rectal malformations) (Figure 5).

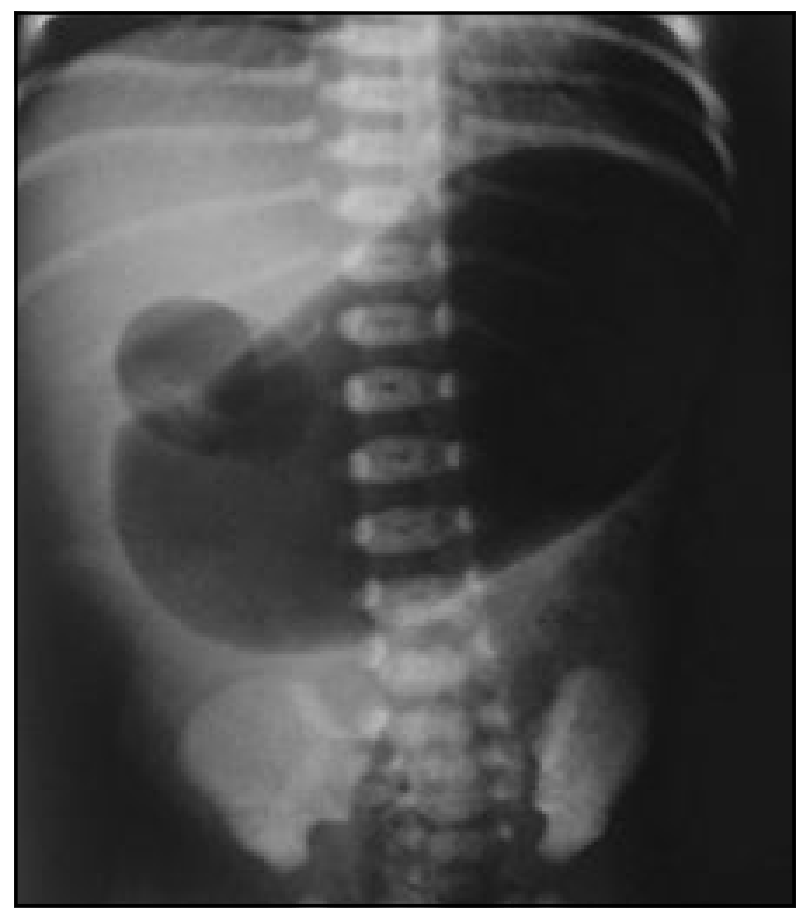

Fig.- 5: Abdominal x ray in Duodenal atresia.

In general the surgery for bowel atresia is relatively straightforward with an anastomosis of proximal to distal bowel tailored depending on the size difference. Complications include delay in feeding with a dilated floppy proximal bowel segment, thus if possible the very dilated proximal segment in small bowel atresia should be excised or trimmed ${ }^{6}$. In duodenal atresia of course this is not an option.

\section{Abdominal wall defects.}

In omphalocoele and gastoschisis the bowel contents can be seen floating freely (gastroschisis) or within a sac (omphalocoele) outside the fetal abdominal cavity. This is fairly easily identified on antenatal ultrasound. This may be an indication for timed delivery and definite consideration of a Caesarean section delivery ${ }^{7}$ to prevent any trauma to the bowel or the sac during delivery. (Figure 6).

Immediate standby by the surgical team with bowel/ sac care is necessary. We clean and wrap in clingwrap to protect the bowel and prevent contamination as well as hypothermia and kinking of vessels and at the same time allowing the neonatologist a period of time to stabilize the baby and prepare for surgical closure - primary or staged. In Omphalocoele other associated malformations are common and must be looked for. In Gastrochisis, the malformation occurs generally in isolation, but there may be bowel 


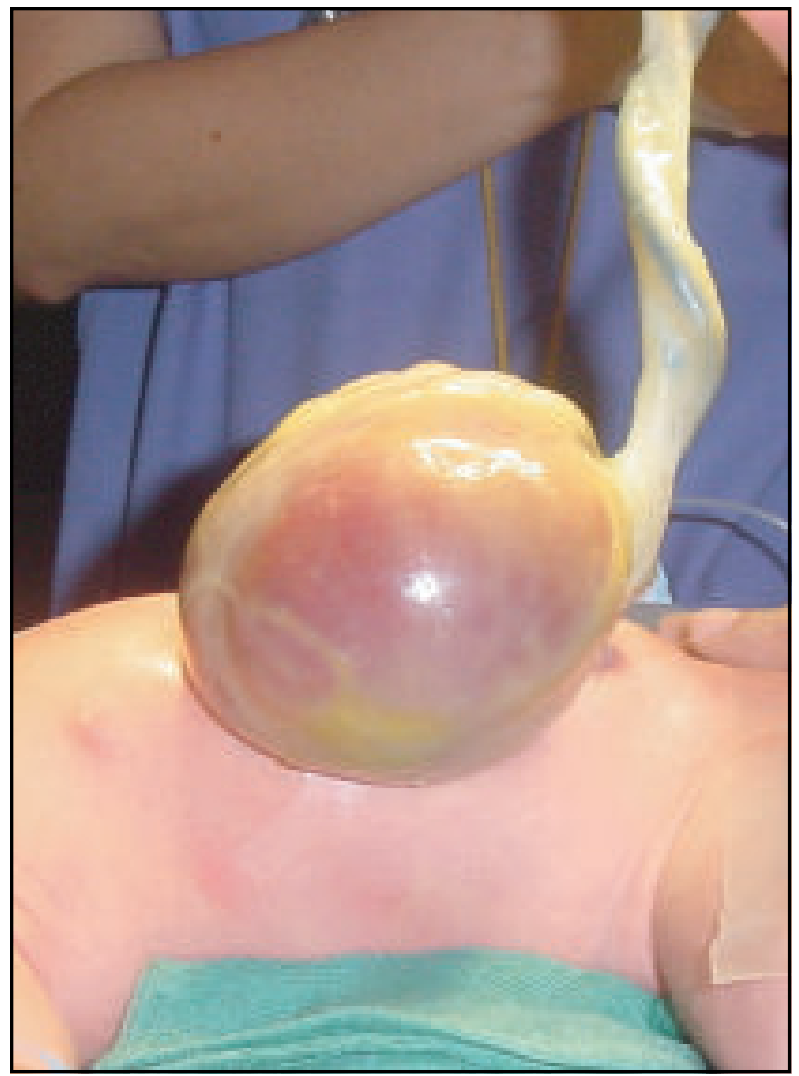

Fig.-6: Omphalocoele with an intact sac. The liver and intestines can be seen under the sac. strictures or atresias associated with the defect. Primary closure gives the best outcomes, but this is not always possible without causing a devastating increase in intra-abdominal pressure with splinting of the diaphragm, so a staged or silo closure is then preferred ${ }^{8,9}$. Mortality in this group of patients is related to sepsis as well as associated malformations. Babies with Gastroschisis may have short bowel.

\section{Outcomes}

In general outcomes after neonatal surgery is continually improving. Mortality in general is related to sepsis and associated malformations. While our numbers are relatively small in Singapore, we are continuing to achieve improved results with increased sophistication in Neonatal and anesthetic care. We prefer to quote our own figures to parents in counseling rather than international textbook figures, as this is more reflective of actual local status. Table-I gives detail of a few of the index neonatal surgical outcomes from KK Hospital.

Table-I

Outcomes of selected Index Surgical conditions from KK Hospital from Jan 2003-Dec 2007

\begin{tabular}{|c|c|c|c|}
\hline Index surgical condition & Number of patients & Outcome & Surgery done \\
\hline \multirow[t]{4}{*}{ Oesophageal atresia } & 14 & Death -1 & Primary repair - 12 \\
\hline & & VACTERL - 4 & \\
\hline & & Congenital Heart - 10 & \\
\hline & & Renal anomalies - 5 & \\
\hline \multirow[t]{3}{*}{ Duodenal atresia } & 8 & Trisomy $21-1$ & Primary repair - All \\
\hline & & Congenital Heart -4 & \\
\hline & & VACTERL - 1 & \\
\hline \multirow[t]{3}{*}{ Small bowel atresia } & 10 & Death - 1 & Primary repair - All \\
\hline & & Congenital heart -3 & \\
\hline & & Multiple atresia - 2 & \\
\hline \multirow[t]{2}{*}{ Omphalocoele } & 11 & Congenital heart -8 & Primary repair - 7 \\
\hline & & Syndromic baby - 4 & Staged repair - 4 \\
\hline \multirow[t]{3}{*}{ Gastroschisis } & 5 & Intestinal atresia - 2 & Primary repair - 3 \\
\hline & & Intestinal failure -1 & Silo repair -2 \\
\hline & & (eventual death) & Multiple surgeries - 1 \\
\hline
\end{tabular}




\section{Summary:}

In neonatal surgical conditions antenatal diagnosis is often possible. This allows for counseling of parents and preparation of the caring team.

Full work up is required for the baby at birth and correct timing of surgery is important.

Surgical outcomes are generally good, the patient can look forward to a full and healthy life.

\section{References:}

1. Raboei EH. The role of the pediatric surgeon in the perinatal multidisciplinary team. Eur J Pediatr Surg. 2008 Oct;18(5):313-7. Epub 2008 Oct 14.

2. Borsellino A et.al. False-positive rate in prenatal diagnosis of surgical anomalies. J Pediatr Surg. 2006 Apr;41(4):826-9.

3. Chiu P, Hedrick HL.Postnatal management and long-term outcome for survivors with congenital diaphragmatic hernia. Prenat Diagn. 2008 Jul;28(7):592-603.

4. Downard CD.Congenital diaphragmatic hernia: an ongoing clinical challenge. Curr Opin Pediatr. 2008 Jun;20(3):300-4.
5. Frenckner BP,et al; Congenital Diaphragmatic Hernia Study Group.16: Prenatal diagnosis of congenital diaphragmatic hernia: how should the babies be delivered? J Pediatr Surg. 2007 Sep;42(9):1533-8.

6. Casaccia G, et al. Congenital intestinal anomalies, neonatal short bowel syndrome, and prenatal/neonatal counseling. J Pediatr Surg. 2006 Apr;41(4):804-7.

7. Hadidi A. et al. Early elective cesarean delivery before 36 weeks vs late spontaneous delivery in infants with gastroschisis. J Pediatr Surg. 2008 Jul;43(7):1342-6.

8. Henrich K, et al. Gastroschisis and omphalocele: treatments and long-term outcomes.

Pediatr Surg Int. 2008 Feb;24(2):167-73. Epub 2007 Nov 6.

9. Fratelli $\mathrm{N}$ et. al. Outcome of antenatally diagnosed abdominal wall defects. Ultrasound Obstet Gynecol. 2007 Sep;30(3):266-70. 